\title{
Self-consistent description of dysprosium isotopes in the doubly midshell region
}

\author{
A. K. Rath, ${ }^{1,2}$ P. D. Stevenson, ${ }^{1}$ P. H. Regan, ${ }^{1}$ F. R. Xu, ${ }^{1,3}$ and P. M. Walker ${ }^{1}$ \\ ${ }^{1}$ Department of Physics, University of Surrey, Guildford GU2 7XH, United Kingdom \\ ${ }^{2}$ Postgraduate Department of Physics, Sambalpur University, Burla 768 019, India \\ ${ }^{3}$ Department of Technical Physics, Peking University, Beijing 100871, China
}

(Received 27 June 2003; published 24 October 2003)

\begin{abstract}
Ground-state deformations, binding energies, and potential energy surfaces have been calculated for eveneven dysprosium isotopes between ${ }^{160} \mathrm{Dy}$ and ${ }^{180} \mathrm{Dy}$ in the framework of density-dependent Hartree-Fock calculations with BCS pairing correlations. Further deformed Hartree-Fock with angular-momentum projection and band-mixing calculations explore the yrast spectra of the nuclides approaching the neutron midshell. Predictions of high- $K$ states in the doubly midshell nucleus ${ }_{66}^{170} \mathrm{Dy}_{104}$ are made.
\end{abstract}

DOI: 10.1103/PhysRevC.68.044315

PACS number(s): 21.10.Dr, 21.10.Re, 21.60.Jz

\section{INTRODUCTION}

The magic numbers $2,8,20,28,50,82$, and 126 are indicators of the shell structure of nuclei. If a nucleus has a magic number of either protons or neutrons then discontinuities will be observed in two-particle separation energies at such systems. When both $N$ and $Z$ correspond to a magic number, the nucleus is deemed to be doubly magic.

By analogy, one can conceive of doubly midshell nuclei, which is to say those in which $N$ and $Z$ lie exactly halfway between consecutive magic numbers. While one might not expect to see a discontinuity in observables across this midshell point, it has been demonstrated that the product of proton and neutron valence particles or holes $N_{p} N_{n}$ outside closed shells correlates monotonically with various collective properties of nuclei, such as the energy of the first $2^{+}$ excited state in even-even nuclei, the energy ratio of the first $4^{+}$to the first $2^{+}$state and the $B\left(E 2: 0_{1}^{+} \rightarrow 2_{1}^{+}\right)$[1]. On the other hand, saturation of $B(E 2)$ values is known to occur with slightly fewer particles than at the midshell for rare-earth nuclei [2], due to microscopic effects beyond the remit of the $N_{p} N_{n}$ scheme.

In our recent Brief Report [3] calculations were made of the properties of the doubly midshell nucleus ${ }_{66}^{170} \mathrm{Dy}_{104}$ using the total Routhian surface (TRS) and configurationconstrained potential energy surface (PES) methods. Based on $N_{p} N_{n}$ systematics, it was suggested that ${ }^{170}$ Dy should be amongst the most quadrupole collective of nuclei with respect to the ground state configuration, since it maximizes $N_{p} N_{n}=16 \times 22=352$ for all nuclei below the doubly-magic ${ }^{208} \mathrm{~Pb}$. The results of the PES calculations in Ref. [3] suggest that ${ }^{170}$ Dy is well deformed in its ground state with $\beta_{2}$ $=0.29$ and $\gamma=0^{\circ}$ with a very stiff potential in both $\beta$ and $\gamma$ degrees of freedom. The TRS results which used a cranked nonaxially deformed Woods Saxon mean field showed a marked constancy in the deformation up to rotational frequencies of at least $\omega=0.3 \mathrm{MeV} / \hbar$. The simultaneous alignment of both protons and neutrons was predicted to occur starting at $\hbar \omega \approx 0.35 \mathrm{MeV}$, corresponding to $I_{x} \approx 14 \hbar$. The deep axially symmetric minimum also led to the suggestion that the $K^{\pi}=6^{+}$two-quasineutron isomer in ${ }^{170} \mathrm{Dy}$ could have a lifetime of the order of hours.
Other recent theoretical work on the excitation spectrum of ${ }^{170} \mathrm{Dy}$ includes tilted axis cranked Hartree-FockBoguliubov (HFB) calculations [4] which predicted a constancy of the quadrupole and triaxial deformations from the ground state up to approximately spin $20 \hbar$. A systematic study using $x$ - and $z$-axis cranked HFB of ${ }^{164-170}$ Dy by Yadav et al. [5] predicts the maximum deformation in Dy isotopes to occur at $N=100\left({ }^{166} \mathrm{Dy}\right)$ and shows a slow change in $\beta$ and $\gamma$ deformation as spin increases.

In this paper we build upon our previous studies [3,4] by exploring isotopes in the region of ${ }^{170} \mathrm{Dy}$ using selfconsistent approaches. In Sec. II, results and discussion of Skyrme-(HF) Hartree-Fock calculations of ground-state properties of dysprosium isotopes from $A=160$ to $A=180$ are presented. Section III details the formalism of the projected HF calculations, the results of which are presented and discussed in Sec. IV. We summarize the work in Sec. V.

\section{SKYRME HARTREE-FOCK CALCULATIONS}

Using a variety of Skyrme parametrizations, HF calculations of dysprosium isotopes between $N=94$ and $N=114$ have been performed. The parametrizations chosen were SIII [6], SkM* [7], SkI4 [8] and SLy4 [9]. SIII has proved capable of describing nuclei in a wide range of the nuclear chart [10]. In common with other early Skyrme parametrizations, SIII was fitted to the properties of spherical, doubly magic nuclei. $\mathrm{SkM}^{*}$ is a modification of a previous force, SkM [11], modified to account for the fission barrier in ${ }^{240} \mathrm{Pu}$. SkI4 adds an extra (isospin) degree of freedom to the spinorbit functional to improve the quality of reproduction of isotope shifts and as such is also a suitable choice for studying isotopic trends. The most recent Skyrme parametrization used here is SLy4, which was fitted to the equation of state of pure neutron matter as well as doubly magic nuclei, and therefore represents a good candidate for calculation of neutron rich systems. The present calculations assumed axial symmetry with and without a constraint on the expectation value of the quadrupole moment, which allows us to view the potential energy surface as a function of $\beta_{2}$, or alternatively allows the local minimum to be found. A unified pairing prescription was used for each interaction consisting of a 


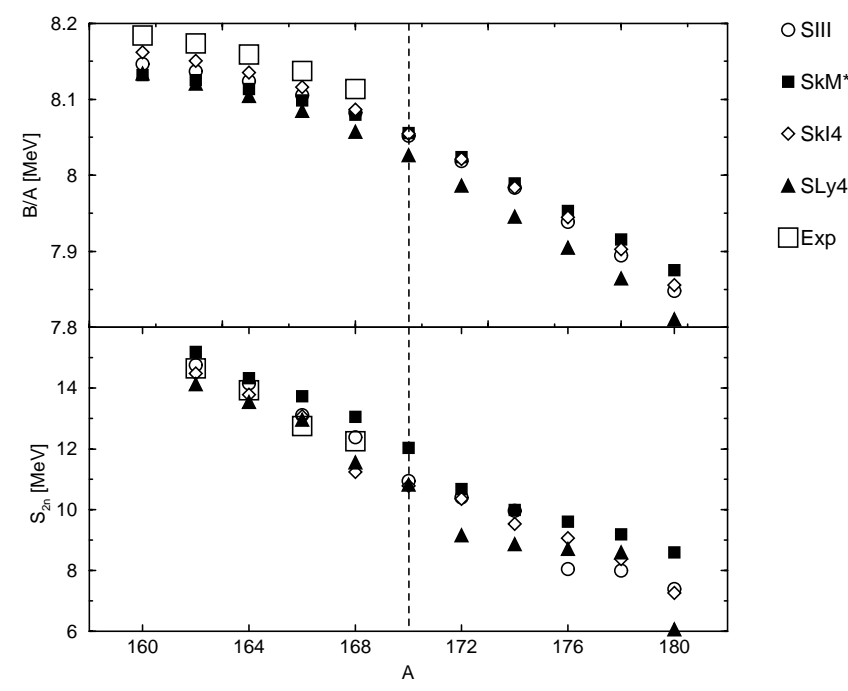

FIG. 1. Binding energy per nucleon and two-neutron separation energy in dysprosium isotopes. The vertical dashed line at $A=170$ indicates the $N=104$ midshell line. Experimental data are from Refs. [15] $(A=160-166)$ and [34] $(A=168)$.

truncated space delta interaction in the BCS approximation. The results of the pairing calculations were also checked using a density-dependent interaction, which has been proposed particularly for use in very neutron rich nuclei $[12,13]$.

The results for each of the Skyrme parametrizations were broadly similar. The binding energy results can be seen in Fig. 1. The upper panel shows the absolute binding energy per nucleon, while the lower panel shows the two-neutron separation energies. The binding energies are seen to be very slightly $(<0.75 \%)$ lower than experimental values, and the observed two-neutron separation energies are closely bracketed by the different Skyrme parametrizations.

Of specific interest in the context of collective behaviour and the potential for long-lived $K$ isomers is the prediction of axially symmetric quadrupole deformations of isotopes of dysprosium in the vicinity of ${ }^{170} \mathrm{Dy}$. The upper panel of Fig. 2 shows the ground-state deformation predicted by the various forces, which are also tabulated in Table I. The central panel shows the energy difference between the local oblate minimum and the global prolate minimum. The lower panel shows the deformation energy, which is the increase in binding of the true deformed ground state compared to a constrained spherical solution. It should be pointed out that these calculations do not include the possibility of triaxial $(\gamma)$ deformation, so what appears as an oblate minimum in these axially deformed calculations is actually a saddle point. Since the nuclei near ${ }^{170}$ Dy are expected to be softer in $\gamma$ than in $\beta$ [3], effects such as shape coexistence and the extent to which the deformation energy is a measure of the robustness of the prolate deformation are not possible to determine from an axially deformed calculation alone. However, as one follows the decreasing of the oblate-prolate energy difference and the deformation energy with increasing $A$, one can attempt to predict when $\beta$ softness will become an important effect.

All the forces show the same general behavior, with the maximum quadrupole deformation occurring in ${ }^{166}$ Dy (SkI4

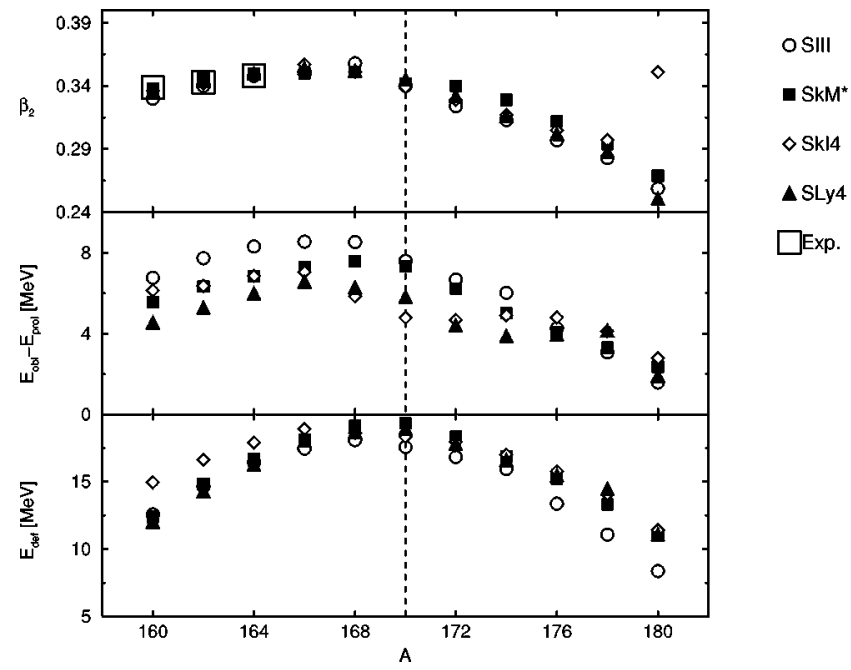

FIG. 2. Static quadrupole deformation $\left(\beta_{2}\right)$, deformation energy and oblate-prolate energy difference in dysprosium isotopes. The vertical dashed line at $A=170$ indicates the $N=104$ midshell line. The apparent anomaly at $A=180$ for SkI4 is due to a double minimum structure appearing in the PES.

and SLy4) or ${ }^{168} \mathrm{Dy}\left(\mathrm{SkM}^{*}\right.$ and SIII). The maximum deformation energy occurs in ${ }^{168} \mathrm{Dy}$ (SkI4 and SIII) or ${ }^{170} \mathrm{Dy}$ $\left(\mathrm{SkM}^{*}\right.$ and SLy4). The difference in energy between the oblate and the prolate minima is largest in either ${ }^{166} \mathrm{Dy}$ (SkI4 and SLy4) or ${ }^{168} \mathrm{Dy}\left(\mathrm{SkM}^{*}\right.$ and SIII). The reliability of the chosen Skyrme parametrizations is confirmed by the excellent agreement between calculated and experimentally derived $\beta_{2}$ values [14].

The different Skyrme forces show only minor differences and one can conclude that, as expected, the even-even dysprosium isotopes between $A=160$ and $A=180$ are all well deformed with $\beta_{2}$ between 0.24 and 0.36 . The largest deformation is reached just before the midshell point, in agreement with recent calculations $[3,15]$ and consistent with the

TABLE I. Experimental first $2^{+}$state and adopted $\beta_{2}$ values. Results are taken from Ref. [14], except for ${ }^{168} \mathrm{Dy}$, whose first $2+$ energy is taken from Ref. [16]. Theoretical ground-state $\beta_{2}$ deformations are from the Skyrme-HF and projected HF calculations. Maximum deformation is indicated by the underlined numbers.

\begin{tabular}{|c|c|c|c|c|c|c|c|}
\hline \multirow[b]{2}{*}{$A$} & \multirow{2}{*}{$\begin{array}{c}E_{2^{+}} \\
\mathrm{keV}\end{array}$} & \multirow{2}{*}{$\begin{array}{c}\beta_{2} \\
\text { Expt }\end{array}$} & \multicolumn{4}{|c|}{$\beta_{2}$ Skyrme forces } & \multirow{2}{*}{$\begin{array}{c}\beta_{2} \\
\text { PHF }\end{array}$} \\
\hline & & & SIII & $\mathrm{SkM}^{*}$ & Sk14 & SLy4 & \\
\hline 160 & 86.79 & 0.339 & 0.330 & 0.338 & 0.336 & 0.336 & 0.245 \\
\hline 162 & 80.66 & 0.343 & 0.340 & 0.347 & 0.342 & 0.344 & 0.254 \\
\hline 164 & 73.39 & 0.348 & 0.348 & 0.350 & 0.349 & 0.350 & 0.260 \\
\hline 166 & 76.58 & & 0.351 & 0.350 & 0.357 & 0.354 & 0.267 \\
\hline 168 & 74.96 & & 0.358 & 0.351 & $\overline{0.351}$ & $\overline{0.352}$ & 0.270 \\
\hline 170 & & & $\overline{0.340}$ & $\overline{0.342}$ & 0.339 & 0.345 & $\overline{0.268}$ \\
\hline 172 & & & 0.324 & 0.340 & 0.329 & 0.332 & \\
\hline 174 & & & 0.313 & 0.329 & 0.317 & 0.316 & \\
\hline 176 & & & 0.297 & 0.312 & 0.305 & 0.302 & \\
\hline 178 & & & 0.283 & 0.294 & 0.297 & 0.288 & \\
\hline 180 & & & 0.259 & 0.269 & 0.351 & 0.337 & \\
\hline
\end{tabular}


experimental finding of $B(E 2)$ saturation around $N=102$ for lower- $Z$ nuclei [16]. The stiffness of the potential well, as measured by the deformation energy reinforces the results of the TRS calculations [3] which suggest a stable deformation up to rather high spin.

Pairing can play an important role in determining deformation, acting as it does towards sphericity. In the case of the nuclei in the present study, increasing the pairing strength within reasonable limits has little effect upon the deformation. The stability of the results was tested against a large (40\%) increase in the pairing strengths in ${ }^{160}$ Dy with SkI4 and found a very small $(\sim 1 \%)$ effect on the deformation. In addition, the density-dependent delta pairing interaction $[10,12]$ was used with the SkI4 force to check that the results were not strongly dependent on the assumed form of the pairing interaction or the details of its strength within reasonable limits. It was found that the predictions for the greatest deformation, deformation energy and oblate-prolate difference and the qualitative isotopic trends were unchanged by the change in pairing interaction.

\section{DEFORMED HARTEE-FOCK CALCULATION AND ANGULAR-MOMENTUM PROJECTION}

To complement the Skyrme HF calculations of the ground states, calculations using HF plus angular-momentum projection (AMP) were performed using a residual interaction to examine excited states. In performing a deformed HartreeFock calculation as a prelude to a shell-model-like mixing of configurations, a substantial part of the residual interaction among nucleons in shell-model orbits is included in the deformed field and one needs only a few particle-hole configurations to obtain an adequate description of the low-energy properties of nuclei.

The axially deformed HF states are expanded in spherical basis states

$$
|\alpha m\rangle=\sum_{j} C_{j m}^{\alpha}|j m\rangle .
$$

The mixing amplitudes of the spherical states form the variational parameters that are solved for in the HF equations by iteration, starting with an appropriate intrinsic state. When a converged solution is obtained, one has the mixing amplitudes for the deformed orbits of protons and neutrons, HF single-particle energies, and the density matrix $\rho$. Various quantities, such as the HF energy, multipole moments, etc., are obtained using the density matrix and the HF singleparticle energies [17].

Prolate HF calculations for the valence nucleons lying outside the ${ }^{132} \mathrm{Sn}$ core were performed for the even-even ${ }^{160-170}$ Dy isotopes using the surface-delta residual interaction $[18,19]$ (with strength $0.3 \mathrm{MeV}$ for $p-p, p-n$, and $n-n$ interactions) within a model space of one major shell each for protons and neutrons. Spherical Nilsson basis states were used. The $3 s_{1 / 2}, 2 d_{3 / 2}, 2 d_{5 / 2}, 1 g_{7 / 2}, 1 h_{9 / 2}$, and $1 h_{11 / 2}$ proton basis states have energies $3.86384,3.56274,1.30468$, $0.60213,7.18459$, and $2.21686 \mathrm{MeV}$, and the $3 p_{1 / 2}, 3 p_{3 / 2}$, $2 f_{5 / 2}, 2 f_{7 / 2}, 1 h_{9 / 2}$, and $1 i_{13 / 2}$ neutron basis states have energies $4.23014,2.84916, \quad 3.31219,0.08990,0.92336$, and $1.71493 \mathrm{MeV}$, respectively.

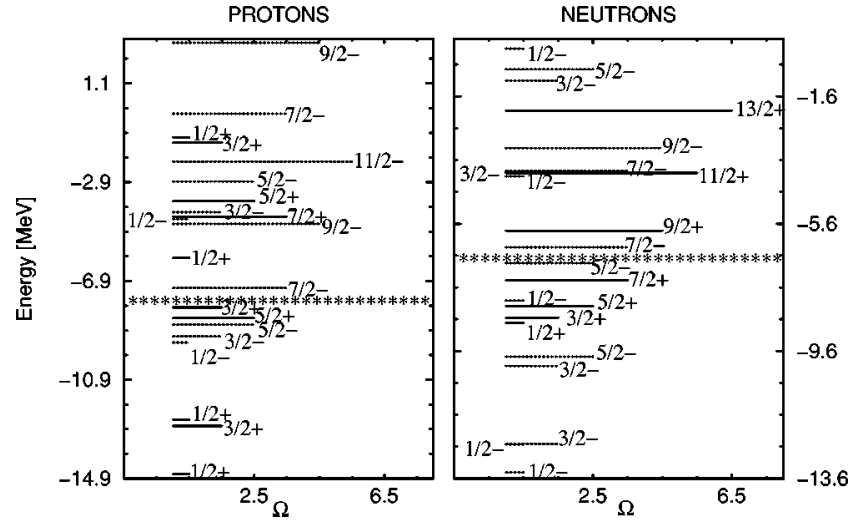

FIG. 3. The prolate Hartree-Fock single-particle orbits for protons and neutrons are shown for ${ }^{170} \mathrm{Dy}$. The time reversed $( \pm \Omega)$ orbits are doubly degenerate. The solid and dashed lines correspond to the positive and negative parity orbits, respectively. The asterisks guide the eye to the Fermi surface which lies amidst high $\Omega$ orbits.

The resulting HF orbits are doubly degenerate and are labeled by the $\Omega$ quantum number, the sum of which for the occupied unpaired orbits gives the $K$ value. Since the Fermi surface is surrounded by large- $\Omega$ orbits for the nuclides under study (see Fig. 3 for the HF orbits for ${ }^{170} \mathrm{Dy}$ ), high- $K$ band structures built upon these single-particle states are likely to be observed. The HF field for these high- $K$ bands (with many large- $\Omega$ excitations) does not possess time reversal symmetry [20].

The HF orbits form a deformed-shell-model basis for the nuclei under consideration, and they contain information about the nuclear structure dynamics and its variation with respect to angular momentum and other important parameters needed for the study of the collective states of the nucleus. Essentially each HF configuration shows the intrinsic distribution of the valence nucleons in the deformedshell-model orbits. For example, the HF configurations shown in Fig. 3 correspond to the ground-state intrinsic configuration in ${ }^{170} \mathrm{Dy}$. For excited states the intrinsic distribution changes and this is taken into account by considering various HF configurations (see Table III) for the study of the yrast and yrare spectra. By appropriate nucleon excitations, the rotation-aligned (RAL) $K=1$ structure and the high- $K$ configurations are selected for angular-momentum projection.

In contrast to the large number $(\approx 100)$ of configurations needed in the projected shell model [21] for understanding the yrast spectra, it is found that angular-momentum projection from a few low-lying HF configurations gives a reasonable description of the yrast structure. This is due to the fact that the residual interaction has been used in the solution of the HF equations, so that the HF single-particle states and the various multiparticle configurations built from them are already closer to the final answer that comes from a full solution of the many-body Schrödinger equation [22]. In fact AMP from a single $K$ configuration reproduces the energy features of the low-lying yrast states with good accuracy (see Fig. 4 for ${ }^{160-168}$ Dy). The current work specifically concentrates on low-lying excitations which give rise to high- $K$ and low- $K$ (RAL) configurations in ${ }^{170} \mathrm{Dy}$. 

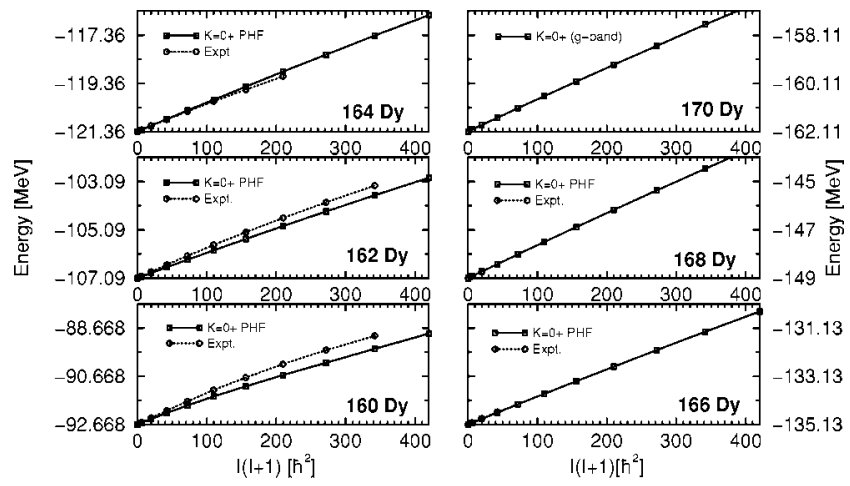

FIG. 4. The PHF results for the ground-state configuration $(K$ $=0+)$ are compared with available experimental data $[15,16]$ for the even-even ${ }^{160-170} \mathrm{Dy}$.

Each HF configuration $|\Phi\rangle_{K}$ is a superposition of states of good angular momentum. These states of good $I$ are projected out by means of the projection operator [23]

$$
P_{K}^{I M}=\frac{2 I+1}{8 \pi^{2}} \int d \Omega D_{M K}^{I}{ }^{*}(\Omega) R(\Omega) .
$$

The Hamiltonian overlap is given by

$$
\begin{aligned}
\left\langle\Psi_{K_{2}}^{I}|H| \Psi_{K_{1}}^{I}\right\rangle= & \frac{2 I+1}{2} \frac{1}{\left(N_{K_{1} K_{1}}^{I} N_{K_{2} K_{2}}^{I}\right)^{1 / 2}} \int d \theta \sin \theta d_{K_{2} K_{1}}^{I}(\theta) \\
& \times\left\langle\phi_{K_{2}}\left|H e^{-i \theta J_{y}}\right| \phi_{K_{1}}\right\rangle
\end{aligned}
$$

with $N_{K K}^{I}=\left\langle\Phi\left|P_{K}^{I K}\right| \Phi\right\rangle$.

Importantly, the angular momentum projection operator (2) restores rotational invariance and the high- $K$ structures are described without any preference being given to the orientation of the rotation axis. Projected HF (PHF) calculations have been shown to give a good understanding of the yrast structure and signature effects in nuclei [24-30].

The energy, $E 2$ and $M 1$ operators, and angular momentum carried by the nucleons $\vec{J}=\left(\vec{J}_{\text {core }}+\vec{J}_{R A L}\right)$ can be evaluated using the following equation:

$$
\begin{aligned}
\left\langle\Psi_{K_{1}}^{I_{1}}\left\|T^{\lambda}\right\| \Psi_{K_{2}}^{I_{2}}\right\rangle= & \frac{\left(2 I_{2}+1\right)\left(2 I_{1}+1\right)^{1 / 2}}{2\left(N_{K_{1} K_{1}}^{I_{1}} N_{K_{2} K_{2}}^{I_{2}}\right)^{1 / 2}} \sum_{\nu} C_{K_{1}-\nu \nu K_{1}}^{I_{2} \lambda I_{1}} \\
& \times \int_{0}^{\pi} d \theta \sin \theta d_{K_{1}-\nu K_{2}}^{I_{2}}(\theta) \\
& \times\left\langle\Phi_{K_{1}}\left|T_{\nu}^{\lambda} e^{-i \theta J_{y}}\right| \Phi_{K_{2}}\right\rangle,
\end{aligned}
$$

where $T^{\lambda}$ is the electromagnetic tensor operator. More details of the formalism can be found in Refs. [24,25,28].

The states $\Psi^{I_{1} M}$ and $\Psi^{I_{2} M}$, with definite angular momenta $I_{1}$ and $I_{2}$ projected from various intrinsic states $\Phi_{K_{1}}$ and $\Phi_{K_{2}}$, are in general not orthogonal to each other even though $\Phi_{K_{1}}$ and $\Phi_{K_{2}}$ are orthogonal before angular-momentum projection. However, if the Hamiltonian is diagonalized in the subspace generated from the projected states, then the resulting eigenstates are orthogonal. One obtains
TABLE II. The values of the quadrupole $\left[Q 2=\left\langle r^{2} Y_{20}(\theta)\right\rangle\right]$ and hexadecapole $\left[Q 4=\left\langle r^{4} Y_{40}(\theta)\right\rangle\right]$ moments obtained for prolate $\mathrm{HF}$ ground states in even-even ${ }^{160-170} \mathrm{Dy}$ are given in units of the harmonic oscillator length parameter, $b\left(=0.9 A^{1 / 3}+0.7 \mathrm{fm}\right)$.

\begin{tabular}{lcccccc}
\hline \hline Nuclei & $K^{\pi}$ & $\begin{array}{c}Q_{2} \\
\text { Protons } \\
\text { in b }^{2}\end{array}$ & $\begin{array}{c}Q_{2} \\
\text { Neutrons } \\
\text { in b }^{2}\end{array}$ & $\beta_{2}$ & $\begin{array}{c}Q_{4} \\
\text { Protons } \\
\text { in b }^{4}\end{array}$ & $\begin{array}{c}Q_{4} \\
\text { Neutrons } \\
\text { in b }\end{array}$ \\
\hline${ }^{160} \mathrm{Dy}$ & $0^{+}$ & 13.839 & 18.4034 & 0.245 & 9.252 & 38.392 \\
${ }^{162} \mathrm{Dy}$ & $0^{+}$ & 13.866 & 20.4676 & 0.254 & 9.419 & 50.806 \\
${ }^{164} \mathrm{Dy}$ & $0^{+}$ & 13.84 & 21.974 & 0.260 & 9.318 & 48.020 \\
${ }^{166} \mathrm{Dy}$ & $0^{+}$ & 13.941 & 23.578 & 0.267 & 8.789 & 28.570 \\
${ }^{168} \mathrm{Dy}$ & $0^{+}$ & 13.955 & 24.193 & 0.270 & 8.456 & 9.779 \\
${ }^{170} \mathrm{Dy}$ & $0^{+}$ & 13.968 & 23.660 & 0.268 & 8.128 & -10.849 \\
\hline \hline
\end{tabular}

$$
\sum_{K^{\prime}}\left(H_{K^{\prime} K}^{I}-E^{I} N_{K^{\prime} K}^{I}\right) b_{K^{\prime}}^{I}=0
$$

Here $H_{K K}^{I}$ and $N_{K K}^{I}$ are the Hamiltonian and amplitude overlaps. The $b$ parameters are the orthonormalized amplitudes:

$$
\Psi^{I M}=\sum_{K} b_{K}^{I} \Psi_{K}^{I M}
$$

With these orthonormalized states, the matrix elements of various tensor operators can be calculated. The mixing of $K$ intrinsic structures can be inferred from the orthonormalized wave functions which are discussed in the following sections.

\section{NEAR YRAST STRUCTURE OF ${ }^{170}$ Dy FROM PHF CALCULATIONS}

\section{A. Ground state properties of ${ }^{160-170} \mathrm{Dy}$}

Prolate deformed HF calculations were performed for ${ }^{160-170}$ Dy. The prolate HF orbits for ${ }^{170} \mathrm{Dy}$ are shown in Fig. 3 . The quadrupole and hexadecapole moments for the prolate solutions are shown for all the nuclei in Table II. For comparison with other calculations, the ground-state $\beta_{2}$ deformations have been extracted and are listed in Table I. Amongst the calculated isotopes, ${ }^{168} \mathrm{Dy}$ was predicted to have the maximum $\beta_{2}$ deformation. This is in qualitative agreement with the results of Sec. II, and with previous calculations $[3,5,31]$ showing that this projected HF approach gives reasonable single-particle structure, which is the main determinant of deformation in a mean-field picture. As such it provides a good starting point for the study of excited states in a fully self-consistent framework. AMP from the ground-state HF configurations for the ${ }^{160-168}$ Dy nuclei gives a reasonable reproduction of the low-lying yrast states (see Fig. 4). The size and variation in the neutron hexadecapole deformation are noticeable in the isotopic chain. It decreases and becomes negative with increasing neutron number $[32,33]$. 
TABLE III. Details of the different intrinsic configurations obtained by excitations of protons and neutrons over the ${ }^{170}$ Dy HF ground state shown in Fig. 3. The yrast and yrare states are obtained by mixing these configurations. The positive and negative numbers given as superscripts of the HF orbits [i.e., $(\Omega)^{n}$ ] represent the number of particles and holes, respectively.

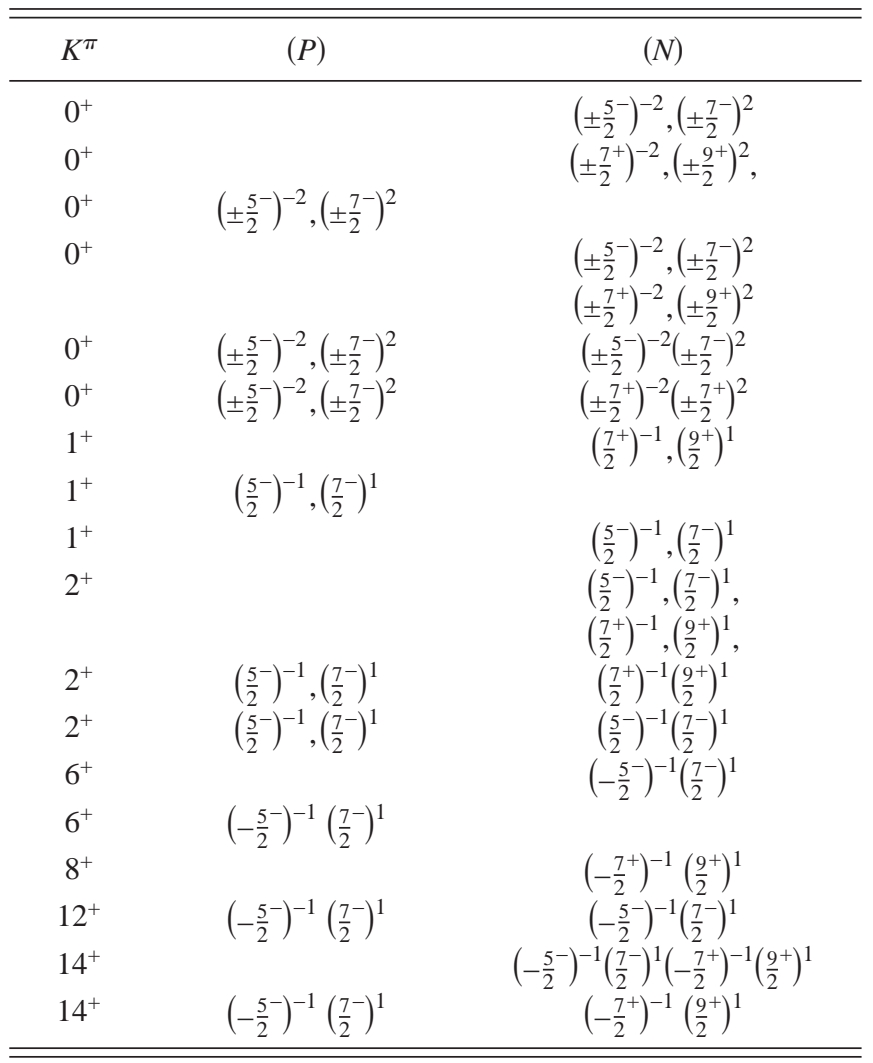

\section{B. Band crossings in ${ }^{170} \mathrm{Dy}$ and band mixing}

Since the $\pi h_{11 / 2}$ and $\nu i_{13 / 2}$ orbits are half filled in 168,170 Dy, these nuclei are found to be well deformed and hence rich rotational band structures are expected.

To find the relative importance of different $K$-intrinsic structures, band-mixing calculations were performed among 19 bands, including the $K=0^{+}$ground band. The intrinsic configurations for the bands were obtained by particle-hole excitations from the HF ground state. These configurations, listed in Table III, can be classified into three types: (i) Low$K$ RAL, (ii) high- $K$ deformation aligned (DAL) and (iii) $K$ $=0,2 p-2 h$ pairing type excitations. For example, considering the states either side of the neutron Fermi surface (see Fig. 3 ), one could have (i) excitation of a neutron from $7 / 2^{+}$to $9 / 2^{+}$giving rise to a $K=1^{+}$RAL structure, (ii) excitation of neutron from $-5 / 2^{-}$to $7 / 2^{-}$giving a high- $K=6^{+}$state, and finally (iii) promotion of a pair of neutrons from $\pm 5 / 2^{-}$to $\pm 7 / 2^{-}$state giving rise to a $K^{\pi}=0^{+}$configuration. Particlehole excitations of the above types (i, ii, and iii) involving the $\nu i_{13 / 2}$ and $\pi h_{11 / 2}$ single-particle states and their linear combinations lead to 18 configurations (six configurations of each type). These were mixed with the ground-state configuration to obtain the yrast and yrare states. Mixing of $K=0$ configurations involving $2 p-2 h$ pairing-type excitations is found to be important in understanding the high spin spec-

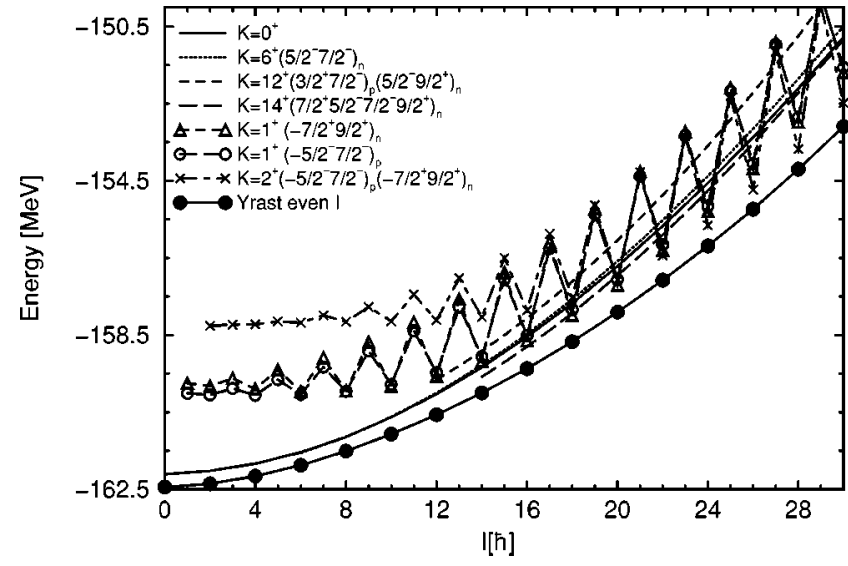

FIG. 5. The PHF results for the low-lying positive parity configurations in ${ }^{170} \mathrm{Dy}$. The yrast states coming from band mixing are also shown. The $\Omega$ values of the unpaired proton/neutrons constituting different $K$ structures are shown against each band.

troscopy in general and excitation energies of the high- $K$ structures in particular. This is one way of accounting for pairing in the HF formalism, preserving the particle number.

It is illuminating to examine the behavior of these various bands before the mixing takes place to get an idea of competition between structures and to more fully appreciate the effects of the mixing. Since ${ }^{170} \mathrm{Dy}$ is well deformed and the unique parity high- $j$ orbits, such as $\pi h_{11 / 2}$ and $\nu i_{13 / 2}$, are approximately half filled, one may be able to see competition between these single-particle orbits to dominate the yrast structure. The $\pi h_{11 / 2}$ shell is exactly half filled with the proton Fermi surface lying in between the $5 / 2^{-}$and $7 / 2^{-}$substates. The neutron Fermi surface is in between $\Omega=7 / 2^{+}$and $9 / 2^{+}$substates of the $i_{13 / 2}$ shell which is slightly more than half filled. Both protons and neutrons can undergo Coriolis mixing and so compete in rotational alignment to cause back bending. One-proton excitation from $5 / 2^{-}$to $7 / 2^{-}$gives rise to a $K=1^{+},\left(\pi h_{11 / 2}\right)^{2}$ rotation-aligned configuration. Similarly, a one-neutron $p$ - $h$ excitation from $7 / 2^{+}$to $9 / 2^{+}$state gives rise to the $K=1^{+},\left(\nu i_{13 / 2}\right)^{2}$ rotation-aligned configuration. Angular-momentum projection from these intrinsic structures gives the corresponding $s$ bands which are easily distinguished by their energy staggering between odd and even spins. These bands along with rotational band structures obtained by AMP from the set of low-lying HF configurations (listed in Table III) are shown in Fig. 5. From the PHF calculations, it is found that both $\pi h_{11 / 2}$ and $\nu i_{13 / 2}$ RAL bands simultaneously compete with the $K^{\pi}=0^{+}$ground band. These RAL bands appear to cross the $K=0$ ground state at around $I=14 \hbar$ and another band crossing is found at around $I$ $=22 \hbar$ when the $K=2^{+}$intrinsic structure with the joint alignment of the $\pi h_{11 / 2}$ and $\nu i_{13 / 2}$ apparently becomes yrast. In the PHF calculation the $K=6^{+}$and $K=14^{+}$bandheads appear to be yrast at $I=6$ and $I=14$, respectively. However, after band mixing, these structures are pushed up in energy and dominate the yrare structure. The details of the high- $K$ band structures are discussed in the following section. The yrast and yrare states obtained after band mixing are shown in Fig. 6 . Figure 7 shows the $K$ distribution in the yrast even-spin states. The band-mixing results indicate that there is indeed a 


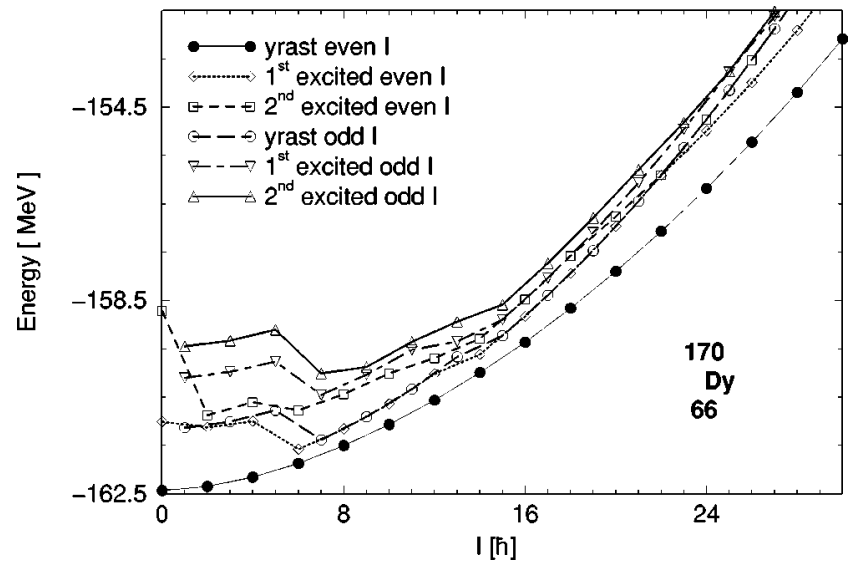

FIG. 6. The yrast and yrare energy spectra obtained after band mixing are shown.

crossing at around $I=14 \hbar$ where the $K=0$ ground-state component of the wave function almost vanishes. The contribution from other $K=0$ components (arising from pair excitations) at the band crossing is nonvanishing. At, and beyond $I=14 \hbar$, the $K=1$ components of both proton and neutron aligned intrinsic structures dominate, with the $i_{13 / 2}$ neutron component larger than the $h_{11 / 2}$ proton. The total low- $K$ ( $K$ $=1,2)$ components dominate the yrast structure above crossing. This total low- $K$ component is itself dominated by the two $K=1$ RAL configurations discussed above, with contributions from the $K=2$ states (not separately shown) giving a much smaller contribution than the $K=1$ structures. The PHF band crossing around $I=22 \hbar$ is therefore suppressed in the band-mixing calculation. At higher spin, the "ground-state" band appears to regain a large part of the amplitude. This is presumably due to an increase in the amplitude of configu-

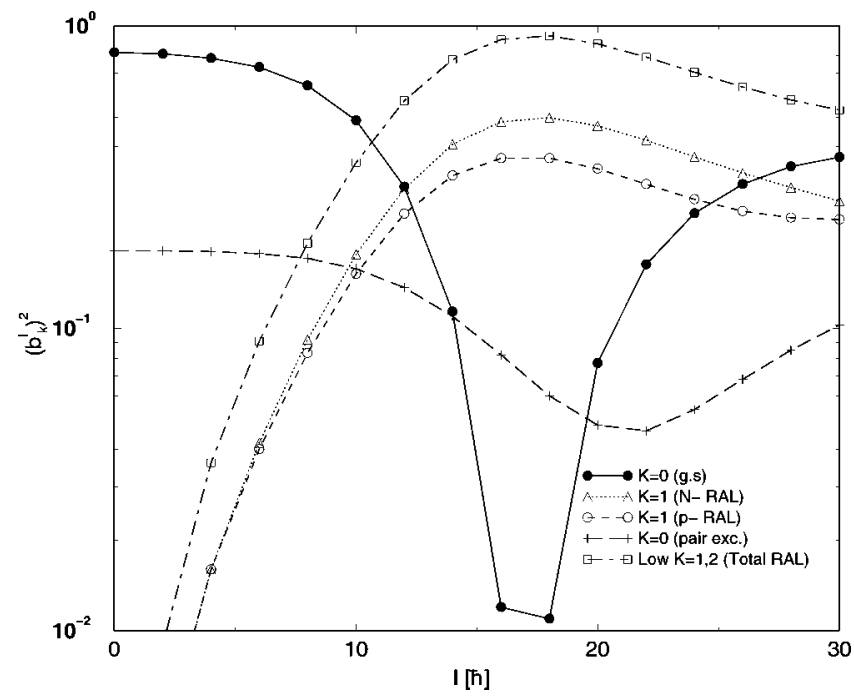

FIG. 7. The $K$ content of the even $I$ yrast states is shown. The competition between different low- $K$ structures is evident. The ground-state $(K=0)$ components almost vanish at band crossing. However the contribution of the other $K=0$ components obtained from pairing type excitations are nonzero. The neutron rotation aligned ( $n$-RAL) structure dominates over the proton aligned structure near crossing $(I=14)$.
TABLE IV. The proton $(\mathrm{P})$ and neutron $(\mathrm{N})$ intrinsic configurations for possible high- $K$ states in ${ }^{170}$ Dy as predicted from PHF and band mixing calculations.

\begin{tabular}{|c|c|c|c|c|}
\hline$K^{\pi}$ & $(P)$ & $(N)$ & $\begin{array}{c}E^{*} \\
\mathrm{PHF} \\
\mathrm{MeV}\end{array}$ & Band mixing \\
\hline $6^{+}$ & & $\frac{5}{2}^{-} \frac{7}{2}^{-}$ & 0.559 & 0.857 \\
\hline $6^{+}$ & $\frac{5}{2}^{-} \frac{7}{2}^{-}$ & & 1.881 & 2.244 \\
\hline $12^{+}$ & $\frac{3}{2}+\frac{7}{2}-$ & $\frac{5}{2}^{-9} \frac{9}{2}^{+}$ & 2.454 & \\
\hline $12^{+}$ & $\frac{5}{2}^{-} \frac{7}{2}^{-}$ & $\frac{5}{2}^{-} \frac{7}{2}^{-}$ & 2.412 & 2.743 \\
\hline $14^{+}$ & & $\frac{5}{2}-\frac{7}{2}-\frac{7}{2}+\frac{9}{2}+$ & 2.524 & 2.817 \\
\hline $14^{+}$ & $\frac{5}{2}^{-} \frac{7}{2}^{-}$ & $\frac{7}{2}+\frac{9}{2}+$ & 3.623 & 3.992 \\
\hline $7^{-}$ & & $\frac{7}{2}+\frac{7}{2}-$ & 1.361 & \\
\hline $7^{-}$ & & $\frac{5}{2} \frac{9}{2}^{+}$ & 1.437 & \\
\hline $5^{-}$ & $\frac{3}{2}+\frac{7-}{2}$ & & 1.159 & \\
\hline $11^{-}$ & $\frac{3}{2}+\frac{7}{2}-$ & $\frac{5}{2}^{-} \frac{7}{2}^{-}$ & 1.620 & \\
\hline $12^{-}$ & $\frac{5}{2}^{+} \frac{7}{2}^{-}$ & $\frac{5}{2}^{-} \frac{7}{2}^{-}$ & 1.952 & \\
\hline $14^{-}$ & $\frac{5}{2}+\frac{7}{2}-$ & $\frac{7}{2} \frac{9}{2}^{+}$ & 3.393 & \\
\hline
\end{tabular}

rations not in the space, but which look more like the fully paired $K=0$ ground state than other available configurations. There is negligible contribution from the high- $K$ configurations in the yrast structure.

It is noted that the gradual and simultaneous increase of the proton and neutron RAL configurations in the yrast states is consistent with the previously presented calculations [3] using a TRS (cranked shell-model) approach, lending credence to both approaches.

\section{High- $K$ structures in ${ }^{170} \mathrm{Dy}$}

Since both proton and neutron Fermi surfaces are surrounded by relatively high- $\Omega$ orbits one expects several high- $K$ states with different intrinsic structures. Only a few low-lying high- $K$ structures, predicted to compete energetically with the yrast states, are concentrated on here. It is found that in PHF calculations the excitation energies of the high- $K$ structures are often underestimated as pairing-type excitations are not explicitly included. It is observed that mixing of various $K=0^{+}$configurations obtained by pairingtype excitations (i.e., $2 p-2 h, 4 p-4 h$ ) leads to a better estimate of the excitation energies. The intrinsic structures and the excitation energies of possible low-lying, high- $K$ states are given in Table IV. A neutron excitation (from $-5 / 2^{-}$to $7 / 2^{-}$) across the Fermi surface gives the lowest $K=6^{+}$structure at an excitation of about $0.85 \mathrm{MeV}$. The other possible $K=6^{+}$ structure has two unpaired protons in the $\Omega^{\pi}=5 / 2^{-}$and $7 / 2^{-}$ orbits, respectively. This one was predicted to lie high in excitation energy and have small (two percent) admixture with the $K=6^{+}$neutron excitation. The rotational band based on the two-neutron $K=6^{+}$state should be favored. The calculations predict four high- $K(\geqslant 10)$ states with different intrinsic structures which appear on and around the band crossing $(I \simeq 14)$. Two $K=12^{+}$intrinsic structures are found at approximately the same excitation energy $(\sim 2.5 \mathrm{MeV})$. 
There are two $K=14^{+}$states, both of which have a fourquasiparticle nature: one with a four unpaired-neutron $\left(5 / 2^{-}\right.$, $7 / 2^{-}, 7 / 2^{+}, 9 / 2^{+}$) structure and the other with two unpaired protons $\left(5 / 2^{-}, 7 / 2^{-}\right)$and two unpaired neutrons $\left(7 / 2^{+}, 9 / 2^{+}\right)$. The first $K=14^{+}$state with a calculated bandhead energy of $2.8 \mathrm{MeV}$ is predicted to be energetically favored compared to the second $K=14^{+}$structure which is calculated to lie $\approx 1.2 \mathrm{MeV}$ higher in excitation energy. Band-mixing calculations indicate that these structures mix very little with each other or with other low- $K$ intrinsic structures, reflecting the robustness of the $K$ quantum number, at least amongst high$K$ states, in this axially symmetric nucleus. Observation of these high- $K$ isomeric states is awaited.

While this study has concentrated on positive parity excited states, negative parity states should also exist in ${ }^{170} \mathrm{Dy}$. Several single-particle orbits near the Fermi surface have opposite parity. For example, the $7 / 2^{+}$neutron state is below the Fermi surface while the $7 / 2^{-}$orbit is just above. Hence the excitation of a neutron between these two orbits gives rise to the lowest $K^{\pi}=7^{-}$configuration, at about $1.3 \mathrm{MeV}$. Several such relatively high- $K$ two-quasiparticle negative parity configurations are also possible. Importantly, a $K=0^{-}$ structure with a $-7 / 2^{+}, 7 / 2^{-}$character is found at about $1.5 \mathrm{MeV}$ and shows energy staggering. The lowest fourquasiparticle negative parity configuration has $K=11$ with a two-quasiproton $\left(3 / 2^{+}, 7 / 2^{-}\right)$and two-quasineutron $\left(5 / 2^{-}, 7 / 2^{-}\right)$structure. A $K^{\pi}=12^{-}$configuration, which differs from the above $K=11^{-}$state by having a proton configuration $\left(5 / 2^{+}, 7 / 2^{-}\right)$, might also compete for yrast status. Some of the possible negative parity $K$ structures are given in Table IV.

In summary, 19 different $K$-intrinsic configurations have been used in band-mixing calculations to find out the relative importance of the different structures. The band-mixing results of the yrast and yrare spectra are shown in Fig. 6. The yrast states are dominated by low- $K$ components, as shown in Fig. 7. The yrare states represent rotational bands based on prominent high- $K$ structures such as $K=6^{+}$and $K=14^{+}$. The first excited states are based on a $K=6^{+}$intrinsic structure up to spin $I=14$. Above this, the states of the $K=14^{+}$band become energetically favored over the $K=6^{+}$band.

\section{CONCLUSION}

Fully self-consistent calculations have been performed for isotopes of dysprosium around the region of the midshell. It is found that different forces and parametrizations used in $\mathrm{HF}+\mathrm{BCS}$ calculations for ground-state properties give qualitatively similar results, with indicators of collectivity generally peaking for $N<104$. There is some spread in results, though the results of the different Skyrme parametrizations are generally close to experiment, where data are available. The ground states of the projected HF calculations are in broad agreement with the no-core Skyrme mean-field calculations and with other studies, supporting the approach that the PHF ground state provides a good starting point for calculations of excited state spectra.

The PHF calculations followed by band mixing predict the existence of several structures, including some high- $K$ states. The band mixing tends to push these states up in energy, to more physically consistent values, compared to the PHF calculation alone. The $K^{\pi}=6^{+}$and $K^{\pi}=14^{+}$states both show very little mixing with other $K$ states, suggesting that $K$ is a good quantum number for these states which may result in very long-lived $K$ isomers, with large experimental hindrance for electromagnetic decay.

\section{ACKNOWLEDGMENTS}

This work was funded by the UK EPSRC. One of the authors (A.K.R.) acknowledges the Association of Commonwealth Universities (UK) (Ref. INCF-2000-61) in collaboration with the University Grants Commission (India). A.K.R. also acknowledges a FIST grant to the Physics department of Sambalpur University by the DST (India).
[1] N. V. Zamfir, R. F. Casten, and D. S. Brenner, Phys. Rev. Lett. 72, 3480 (1994)

[2] R. F. Casten, K. Heyde, and A. Wolf, Phys. Lett. B 208, 33 (1988).

[3] P. H. Regan, F. R. Xu, P. M. Walker, M. Oi, A. K. Rath, and P. D. Stevenson, Phys. Rev. C 65, 037302 (2002).

[4] M. Oi, P. H. Regan, F. R. Xu, P. M. Walker, and P. D. Stevenson, Prog. Theor. Phys. Suppl. 146, 609 (2002).

[5] H. L. Yadav, M. Kaushik, I. R. Jakhar, and A. Ansari, Part. Nucl., Lett. 112, 66 (2002).

[6] M. Beiner, H. Flocard, N. Van Giai, and P. Quentin, Nucl. Phys. A361, 29 (1975).

[7] J. Bartel, P. Quentin, M. Brack, C. Guet, and H.-B. Hakansson, Nucl. Phys. A386, 79 (1982).

[8] P.-G. Reinhard and H. Flocard, Nucl. Phys. A584, 467 (1995).

[9] E. Chabanat, P. Bonche, P. Haensel, J. Meyer, and R. Schaef- fer, Nucl. Phys. A635, 231 (1998).

[10] N. Tajima, S. Takahara, and N. Onishi, Nucl. Phys. A603, 23 (1996).

[11] H. Krivine, J. Treiner, and O. Bohigas, Nucl. Phys. A336, 195 (1980).

[12] M. Bender, K. Rutz, P.-G. Reinhard, and J. A. Maruhn, Eur. Phys. J. A 8, 59 (2000).

[13] N. Tajima, P. Bonche, H. Flocard, P.-H. Heenen, and M. S. Weiss, Nucl. Phys. A551, 434 (1993).

[14] S. Raman, C. W. Nestor, Jr., and P. Tikkanen, At. Data Nucl. Data Tables 78, 1 (2001).

[15] P. Möller, J. R. Nix, W. D. Myers, and W. J. Swiatecki, At. Data Nucl. Data Tables 59, 185 (1995).

[16] M. Asai, S. Ichikawa, K. Tsukada, M. Sakama, M. Shibata, Y. Kojima, A. Osa, I. Nishinaka, Y. Nagame, K. Kawade, and T. Tachibana, Phys. Rev. C 59, 3060 (1999).

[17] G. Ripka, in Advances in Nuclear Physics, edited by M. 
Baranger and E. Vogt (Plenum, New York, 1968), Vol. 1, p. 183.

[18] A. Faessler, P. Plastino, and S. A. Moszkowski, Phys. Rev. 156, 1064 (1967).

[19] P. J. Brussard and P. W. M. Glaudemans, Shell Model Applications in Nuclear Spectroscopy (North Holland, Amsterdam, 1977).

[20] C. R. Praharaj Phys. Rev. Lett. 45, 1238 (1980).

[21] J. A. Sheikh, Y. Sun, and P. M. Walker, Phys. Rev. C 57, R26 (1998).

[22] S. B. Khadkikar, S. C. K. Nair, and S. P. Pandya, Phys. Lett. 36B, 290 (1971).

[23] R. E. Peierls and J. Yoccoz, Proc. Roy. Phys. Soc. A70, 381 (1957).

[24] A. K. Rath, C. R. Praharaj, and S. B. Khadkikar, Phys. Rev. C
47, 1990 (1993).

[25] J. A. Sheikh, A. K. Rath, and C. R. Praharaj, J. Phys. G 16, L275 (1990).

[26] A. K. Rath and C. R. Praharaj, Pramana 35, L405 (1990).

[27] A. K. Rath and C. R. Praharaj, Pramana 36, L125 (1991).

[28] C. R. Praharaj, Phys. Lett. 119B, 17 (1982).

[29] C. R. Praharaj, J. Phys. G 14, 843 (1988).

[30] C. R. Praharaj and A. K. Rath, Europhys. Lett. 7, 305 (1988).

[31] G. A. Lalazissis, M. M. Sharma, and P. Ring, Nucl. Phys. A597 35 (1996).

[32] W. Nazarewicz and P. Rozmej, Nucl. Phys. A369, 396 (1981).

[33] S. K. Patra, S. Yoshida, N. Takigawa, C. R. Praharaj, and A. K. Rath, Phys. Rev. C 51, 2248 (1995).

[34] Xiuqin Lu, Jiyu Zhao, Yehau Cheng, Yong Ma, Zhichang Li, Shuyuan Li, and Ming Ruan, Eur. Phys. J. A 2, 149 (1998). 